\title{
Reviewing Major Mechanisms of $\beta$-Cell Regeneration: A Prospective Treatment for Diabetes Mellitus
}

\author{
Eileen Jin ${ }^{1}$, Emma Djabali ${ }^{1}$ b, Farinoosh Dadrass ${ }^{1}{ }^{c}$, Erin Hannon ${ }^{1}{ }^{d}$ \\ ${ }^{1}$ Georgetown University School of Medicine \\ Keywords: $\alpha$-cells, transdifferentiation, $\beta$-cell duplication, neogenesis, stem cells \\ https://doi.org/10.52504/001c.12643
}

\section{Georgetown Medical Review}

Vol. 4, Issue 1, 2020

\begin{abstract}
Regeneration of human pancreatic $\beta$ cells has the direct ability to treat type 1 and type 2 diabetes mellitus because important characteristics of diabetes include compromised function and/or reduced mass of $\beta$ cells. While there has been limited success in transplanting pancreatic islets to supplement $\beta$ cells in patients with diabetes, the low supply of donors requires a continuation of the search for sustainable sources of $\beta$ cells. Research investigating different mechanisms of $\beta$ cell regeneration has been promising. First, neogenesis of $\beta$ cells in vivo can be conducted by precisely differentiating embryonic stem cells and induced pluripotent stem cells. Second, duplication of $\beta$ cells occurs in vivo but significantly slows down after infancy. Studies using animal models have suggested ways to induce $\beta$-cell duplication in the adult pancreas. Third, the potential to induce endogenous transdifferentiation of other mature pancreatic cells into $\beta$ cells has recently attracted attention, especially in research involving the use of $\alpha$ cells as progenitor cells. This review summarizes the 3 major mechanisms through which $\beta$ cells are regenerated and discusses the challenges associated with translating this research into clinical therapeutics for patients with diabetes. Furthermore, current findings suggest that transdifferentiation of existing pancreatic progenitor cells has the most potential as a source of $\beta$ cells in this field of regenerative medicine.
\end{abstract}

\section{Introduction}

Diabetes mellitus is the most common chronic metabolic disorder, affecting approximately 422 million individuals worldwide. ${ }^{1}$ It is characterized by hyperglycemia due to defects in insulin secretion, action, or both. Several pathogenic processes are involved in diabetes. $\beta$ cells are highly specialized endocrine cells found in the pancreatic islets of Langerhans. These cells sense glucose plasma concentrations and respond by producing and secreting insulin to maintain glucose homeostasis. Type 1 diabetes mellitus is characterized by the autoimmune destruction of the functional $\beta$ cells of the pancreas, causing insulin insufficiency. ${ }^{1}$ Patients with type 2 diabetes initially develop a resistance to insulin in response to the excessive secretion of insulin due to hyperglycemia, which may result in secretory defects or the destruction of $\beta$ cells. ${ }^{1}$ In patients with type 1 and type 2 diabetes, endogenous mechanisms of glycemic control

\footnotetext{
a Authors contributed equally to this work.

b Authors contributed equally to this work.

c Authors contributed equally to this work.

d Authors contributed equally to this work.
} 
are compromised. This compromise can be life-threatening and can lead to long-term complications such as cardiovascular disease, diabetic nephropathy, and diabetic retinopathy. ${ }^{1}$ Therefore, a cure is in high demand.

Pharmacological interventions, such as insulin and/or metformin therapy, and lifestyle changes, such as diet, exercise, and weight loss, are the current treatments for diabetes. ${ }^{1}$ However, these treatments only manage symptoms and do not address the underlying pathology. Alternatively, islet transplantation has been achieved using human cadaver donors to replace $\beta$ cells in patients with diabetes. ${ }^{2}$ Furthermore, islet transplantation has been shown to provide better glucose homeostasis than pharmacological insulin therapy. ${ }^{3}$ Nevertheless, the limited number of donors and recipients' need for lifelong immunosuppressant therapy challenge the potential for the widespread clinical application of islet transplantation in patients with diabetes.

In 2010, it was observed that preterminal clinical conditions of the donor had an effect on the rate of $\beta$-cell replication prior to transplantation. ${ }^{4}$ Donors who were on prolonged life support (mechanical respiration $\geq 3$ days), had kidney dysfunction (serum creatinine $\geq 150 \mu \mathrm{mol} / \mathrm{l}$ ), or were of young age ( $\leq 25$ years) in particular were noted to have significantly higher levels of $\beta$-cell replication, suggesting they were better candidates for islet donation. ${ }^{4}$ The variation of $\beta$ cell replication rates among different patients is interesting because it suggests an endogenous plasticity of $\beta$-cell count. The ability to manipulate the processes by which $\beta$-cells are developed and maintained motivates research in the field of pancreatic regeneration as a means of achieving a potential treatment for diabetes.

Current research in pancreatic $\beta$-cell regeneration is investigating 3 mechanisms: (1) neogenesis via stem cells, (2) the duplication of existing $\beta$ cells, and (3) transdifferentiation of existing pancreatic progenitor cells (Table 1). In this review, we define these mechanisms and analyze their potential use as diabetes therapies. So far, research has pointed towards $\beta$-cell regeneration as optimal for patients with diabetes mellitus because it could provide years of long-term treatment.

\section{Neogenesis of $\beta$ Cells via Stem Cells}

The neogenesis of functional pancreatic $\beta$ cells is defined as the generation of cells that meet the following criteria: (1) the cells express insulin, (2) the cells secrete insulin in response to a glucose challenge, and (3) the cells delaminate and migrate from nonpancreatic tissue to join existing pancreatic islets of Langerhans. ${ }^{5}$ It is important to note that the parameters by which researchers deduce $\beta$-cell neogenesis can vary among studies. For example, some studies have delineated the rapid kinetics of $\beta$-cell insulin secretion as a marker of successful pancreatic $\beta$-cell neogenesis from stem cells, whereas others have examined human $\mathrm{C}$-peptide concentrations and in vivo glucose responsiveness 
Table I: Summary of $\beta$-cell regeneration mechanisms and their application to diabetic patients.

\begin{tabular}{|c|c|c|}
\hline $\begin{array}{l}\text { Mechanism of } \beta \text {-cell } \\
\text { Regeneration }\end{array}$ & Advantages & Disadvantages \\
\hline $\begin{array}{l}\text { Neogenesis of } \beta \text {-cells } \\
\text { from } E_{S C} s^{a} \text { and iPSCs }{ }^{b}\end{array}$ & - Successfully demonstrated in vivo using animal models ${ }^{5-10}$ & $\begin{array}{l}\text { - Ethical qualms }(\mathrm{ESCs})^{2,5} \\
\text { - Immune response }(\mathrm{ESCs})^{2,7} \\
\text { - } \text { Tumorigenesis risks }(\mathrm{ESCs} \text { and } \\
\text { iPSCs) } \\
\text { - } \text { Complex differentiation }^{2,9} \\
\text { protocols } \\
2,6,9-11\end{array}$ \\
\hline $\begin{array}{l}\text { Duplication of residual } \beta \text { - } \\
\text { cells }\end{array}$ & $\begin{array}{l}\text { - Occurs endogenously }{ }^{12-15} \\
\text { - Theoretically, no adverse immune response }\end{array}$ & $\begin{array}{l}\text { - Slows with age }{ }^{14-16} \\
\text { - Risk of apoptosis if over- } \\
\text { stimulated }{ }^{14} \\
\text { - Glucose stimulation may be } \\
\text { contraindicated in hyperglycemic } \\
\text { diabetics }{ }^{1,16,17} \\
\text { - } \text { Lack of residual } \beta \text {-cells in many } \\
\text { diabetics }^{13}\end{array}$ \\
\hline $\begin{array}{l}\text { Transdifferentiation of } \\
\text { other pancreatic } \\
\text { progenitor cells }\end{array}$ & $\begin{array}{l}\text { - Potential for unlimited source of } \beta \text {-cells } 2,5,18-20 \\
\text { - GABA signaling, associated with enhancement of } \alpha \text { - to } \beta \text {-cell } \\
\text { transdifferentiation, has therapeutics already approved for safe } \\
\text { use in humans }{ }^{21-26}\end{array}$ & $\begin{array}{l}\text { - In vivo studies not yet conducted } \\
\text { in humans } \\
\text { - Unclear if epigenetics or } \\
\text { molecular signaling plays more } \\
\text { significant role } 20,27-29\end{array}$ \\
\hline yonic Stem Cells & & \\
\hline
\end{tabular}

to come to similar conclusions. ${ }^{6,7}$ This variation can impact the significance of the results, namely, whether regeneration of bona fide $\beta$ cells has been achieved via stem cell neogenesis or another mechanism. ${ }^{5}$

Stem cell therapy to achieve pancreatic neogenesis is a field that aims to replace destroyed pancreatic $\beta$ cells in both patients with type 1 and type 2 diabetes. Different types of stem cells have been used for pancreatic $\beta$-cell neogenesis, including embryonic stem cells (ESCs) and induced pluripotent stem cells (iPSCs). ${ }^{6}$ Neogenesis using both types of stem cells constitutes an exciting path to address the shortage of donor islets, but it presents some obstacles to potential future clinical applications for the treatment of diabetes. ${ }^{2}$ The use of ESCs raises ethical qualms, requires either combination therapy with immunosuppressants or complex encapsulation techniques, and poses tumorigenesis risks. ${ }^{2} \mathrm{Kim}$ and $\mathrm{Lee}^{2}$ stated that iPSCs, on the other hand, do not present the same ethical issues as do ESCs. This is because iPSCs can be harvested from adult cells, such as skin fibroblasts, whereas ESC harvesting requires the removal of the inner cell mass and termination of the human embryo. Additionally, iPSCs are generated by reprogramming somatic cells obtained from the recipient; therefore, their identical genetic makeup avoids the need for immunosuppressants. Here we also outline the origin of iPSCs and ESCs, along with 2 recent significant studies showing successful in vivo neogenesis of pancreatic $\beta$ cells derived from both types of stem cells. 
ESCs were first generated in 1998 by Thomson et al ${ }^{8}$ using human blastocysts. The researchers derived 5 lines with the ability to maintain the developmental potential to form the 3 embryonic germ layers. Vegas et $\mathrm{al}^{7}$ aimed to isolate the transplanted ESC-generated $\beta$ cells to avoid mounting an immune response against the transplanted $\beta$ cells in recipients using a modified alginate encapsulation sphere. Their research determined an ideal-sized sphere capable of eluding strong innate immune-mediated foreign-body responses in rodents. Furthermore, the application of encapsulated ESC-generated $\beta$ cells was successful in achieving glycemic control in diabetic rodents over 6 months. ${ }^{8}$ To our knowledge, this was the longest successful demonstration of glycemic control using human ESCs in an animal model. Further research must be conducted to evaluate the safety of long-term application of encapsulation therapies before such studies are conducted in humans.

Human iPSCs can be generated from human somatic cells via complex extrinsic signaling pathways involving specific transcription factors. ${ }^{11}$ Takahashi et $a^{11}$ first determined this using skin fibroblasts. They demonstrated that the following 4 transcription factors are sufficient to induce reprogramming of somatic cells into pluripotent stem cells: SOX2, OCT3/4, KLF4, and c-MYC. ${ }^{11}$ Since this groundbreaking initial discovery, many have sought to further research in the field of iPSCs in the hopes of achieving clinically effective cell-replacement therapies.

The translation of iPSC research into therapeutics has been limited by safety risks, for example, tumorigenesis has been associated with the use of reprogramming vectors. ${ }^{9}$ El Khatib et $\mathrm{al}^{9}$ reported that injections of somatic iPSCs reprogrammed by lentiviral vectors to express SOX2, OCT4, KLF4, and c-MYC led to the induction of invasive teratocarcinoma-like tumors in $90 \%$ of observed mice. Tumorigenesis in vector-reprogrammed iPSCs is underlaid by the overexpression of the oncogene $c-M y c$ and presence of residual undifferentiated pluripotent cells. ${ }^{9}$ El Khatib et $\mathrm{al}^{9}$ reported that residual undifferentiated stem cells and iPSCs that overexpressed c-MYC were overly sensitive to trypsin dissociation, whereas iPSC-derived pancreatic endoderm cells were more resistant. They used trypsin to eliminate stem cells at risk of tumorigenesis prior to administration in patients. Concurrently, new reprogramming technologies enabled El Khatib et $\mathrm{al}^{9}$ to generate iPSCs devoid of genomic modification. They also showed that tumorigenesis risks can be avoided by using these transgene-free, c-MYC-independent iPSCs. By combining the use of trypsin to dissociate residual stem cells and the use of transgene-free $\beta$ cells, they successfully transplanted mice with tumor-free pancreatic endoderm cells from iPSCs. ${ }^{9}$ This study in mice elucidates methods by which tumorigenesis risks can be managed in stem cell therapies in a clinical setting. Despite managing the tumorigenesis risks, the iPSC-derived cells did not show proper glucose-responsive C-peptide secretion 3 months after transplantation, thereby demonstrating further need for improved methods of $\beta$-cell differentiation and maturation.? 
A previously conducted study from 2009 successfully generated and transplanted in vitro-matured pancreatic insulin-producing cells from human ESCs and iPSCs into diabetic immunodeficient mice. ${ }^{10}$ Zhang et al ${ }^{10}$ generated human iPSCs using 3 of the 4 reprogramming genes (Sox2, Oct4, and $K l f 4$ ), excluding $c-M y c$ to avoid an increased risk of tumorigenesis. The ESCs and iPSCs were then differentiated into pancreatic cells using a novel stepwise protocol. First, cells were treated with activin A and wortmannin to induce endoderm formation. The differentiated endoderm cells were then treated with retinoic acid, NOGGIN, and FGF7 to induce pancreatic specialization. Lastly, a cocktail of factors known to induce the maturation of insulin-producing cells was used. The newly differentiated cells were then screened for $\beta$-cell-specific markers such as Nkx6-1, Pdx1, MafA, Glut2, and insulin. ${ }^{10}$

These markers were aimed at demonstrating that the cells generated had similar markers to that of mature in vivo pancreatic $\beta$ cells. Zhang et a ${ }^{10}$ demonstrated that the ESC- and iPSC-derived mature insulin-secreting cells released Cpeptide in response to glucose stimulation in a manner like that of an adult human islet cell. Furthermore, this study established a particularly novel and efficient differentiation method: $25 \%$ of cells produced from ESCs were insulin positive. ${ }^{10}$ However, the researchers failed to accurately quantify the percentage of insulin-positive cells generated from iPSCs.

Overall, iPSCs present fewer barriers than ESCs to potential clinical applications in treating diabetes in the future. ESCs present both ethical and immunological obstacles, which iPSCs do not. However, methods for differentiation of both iPSCs and ESCs into $\beta$ cells are complex and timeconsuming and pose significant risks of tumorigenesis. ${ }^{2}$ Until now, these limitations have compromised the application of these therapies for the treatment of patients with diabetes and destroyed $\beta$ cells.

\section{Duplication of Existing $\beta$ Cells}

Multiple studies recently have confirmed that self-duplication of existing $\beta$ cells plays a major role in maintenance and expansion of the postnatal pancreatic $\beta$ cell population. ${ }^{12-14}$ The milestone study in 2004 by Dor et $\mathrm{al}^{12}$ used genetic lineage tracing to determine that new $\beta$ cells are derived from existing ones. Fully differentiated $\beta$ cells in mice were labeled with a genetic marker and reexamined for multiple periods up to 12 months. Newly formed cells were only genetically marked if they originated from the initially labeled cells. As the number of $\beta$ cells increased, it was observed that the percentage of cells with the genetic marker remained constant, indicating that the originally marked cells were the source of new $\beta$ cells. ${ }^{12}$ In addition, a study in 2007 by Teta et $\mathrm{al}^{13}$ not only confirmed that the $\beta$ cells in mice were self-replicating, but also reported a lack of involvement of other specific progenitor cells such as what is observed in the regeneration of the gastrointestinal tract. These findings regarding the duplication of existing $\beta$ cells in the mature pancreas have been extended to humans and were validated by Meier et $\mathrm{al}^{15}$ in 2008 . Their research analyzed 
pancreatic tissue from autopsies in humans between the ages of 2 weeks and 21 years and reported that increased postnatal $\beta$-cell mass was correlated with increased self-duplication. ${ }^{15}$ Additionally, Meier et $\mathrm{al}^{15}$ observed that while islets increased in size, they did not increase in number. This suggests that $\beta$ cell regeneration is not likely due to formation of new islets from endocrine progenitor cells found in the exocrine ductal epithelium. Instead, Meier et $\mathrm{al}^{15}$ concluded that $\beta$ cells are primarily regenerated by means of duplication in postnatal humans.

The endogenous process of $\beta$-cell duplication provides insight into how $\beta$-cell regeneration may be induced in patients who have depleted $\beta$-cell populations; thus, many studies have attempted to characterize this mechanism. In healthy humans, $\beta$ cells are homogenous in their ability to contribute to an increase in the number of $\beta$ cells. ${ }^{15}$ Meier et al ${ }^{15}$ also observed that replication of existing $\beta$ cells is most remarkable during infancy and significantly decreases with age.

Systemic factors, rather than local factors, control the rate of $\beta$-cell duplication. ${ }^{17}$ Unsurprisingly, Porat et $\mathrm{al}^{17}$ found that glucose was a major factor that provoked a mitogenic effect. These findings correlate with the normal physiological negative feedback loop: high blood glucose stimulation of $\beta$-cell duplication could lead to increased insulin secretion and a reduction in blood glucose as glucose is mobilized into cells. More specifically, the study observed that the rate of duplication within $\beta$ cells was controlled by both glycolysis and the subsequent depolarization of the cell membrane due to the closing of ATP-sensitive potassium channels. ${ }^{17}$ The researchers also noticed an increase in $\beta$-cell duplication when glucokinase activators were administered. It is understood that glucokinase activity increases during normal glucose metabolism. ${ }^{17}$ Porat et $\mathrm{al}^{17}$ noted these findings as especially positive in light of the current development of glucokinase activators as drugs to control blood glucose; they highlighted a possible dual-functional treatment to tackle both hyperglycemia and decreased $\beta$-cell population in patients with diabetes.

Studying the cell cycle of $\beta$ cells and the mitogens that influence it has also been useful in further understanding the process of $\beta$-cell duplication. In 2014, Hija et $\mathrm{al}^{16}$ used a mouse model and discovered that $\beta$ cells in the resting phase, G0, required 8 hours of glucose stimulation to enter G1 and commit to the cell cycle. Therefore, the researchers concluded that the length of exposure to glucose is one component in the regulation of the fate of $\beta$ cells, namely, it influences the determination of whether $\beta$ cells secrete insulin or replicate. They also reported that cell age influences the $\beta$ cells' potential to replicate. The understanding that older $\beta$ cells have a decreased likelihood of replication was further supported by observations of older $\beta$ cells requiring a longer period of mitogenic stimulation before committing to the cell cycle. ${ }^{16}$ For example, 6-month-old $\beta$ cells from mice took 13 hours of glucose stimulation to commit to the cell cycle, whereas 5 -week-old $\beta$ cells required only 8 hours of glucose stimulation. 16 The influence of varying durations of glucose stimulation on 
$\beta$-cell duplication is significant because it potentially explains why the $\beta$-cell duplication rate decreases so significantly after infancy in a healthy pancreas. In a healthy individual with sufficient $\beta$-cell secretion of insulin, the spike in glucose after eating is typically cleared in 1 to 2 hours. ${ }^{16}$ Thus, cell-cycle commitment in response to long-term glucose stimulation is a rare occurrence in this context. With age, it becomes even more difficult to induce $\beta$-cell duplication as time requirements for glucose stimulation increase.

In the search for effective mechanisms of $\beta$-cell regeneration, the selfduplication of existing $\beta$ cells within the pancreas is an appealing method, but producing these desirable effects is not without serious hurdles. Glucose overload has a harmful effect on $\beta$ cells, which is one reason it is closely monitored in patients with diabetes. ${ }^{1}$ As previously mentioned, glucose is also a major mitogenic factor for $\beta$-cell proliferation. ${ }^{16,17}$ The necessary management of glucose homeostasis in patients with diabetes may conflict with the potential for increased glucose to induce proliferation effects on $\beta$ cells. Further study of these opposing effects must be undertaken to find a glucose balance that maximizes proliferation while remaining within a healthy range for patients.

A larger concern with stimulating existing $\beta$-cell duplication is the increased possibility for cell apoptosis. ${ }^{14}$ Forced stimulation of a cell to undergo mitosis can trigger cell death. ${ }^{14}$ This paradoxical relationship between increased replication and increased risk of apoptosis could potentially be the underlying cause for the similarly clashing effects of glucose. For instance, glucose might initially cause a mitogenic effect, but repeated high exposure to glucose would activate protective mechanisms and induce cell death. While future directions for evaluating the potential of $\beta$-cell self-duplication may uncover ways to mitigate apoptosis, a complete lack of cell division regulation could lead to tumorigenesis and, therefore, should be avoided.

Further, duplication of existing $\beta$ cells occurs very slowly, and this problem is exacerbated in patients with diabetes because of their decreased number of healthy $\beta$ cells. ${ }^{3}$ Therefore, patients who are most suited for a treatment inducing $\beta$-cell replication are those experiencing the initial onset of diabetes and who still have residual functioning $\beta$ cells. Perhaps individuals who are at a high risk of developing diabetes may also consider inducing $\beta$-cell duplication as a preventive measure. There are challenges associated with the application of $\beta$-cell duplication for the treatment of diabetes, including the slow rate of duplication, the current risk of apoptosis when overstimulated, and the low number of $\beta$ cells in patients with diabetes. Therefore, $\beta$-cell duplication may need to be combined with another method of $\beta$-cell regeneration for clinical application in effectively managing diabetes. 


\section{Transdifferentiation of Other Pancreatic Precursor Cells}

Transdifferentiation is the reversion of mature cells to a progenitor cell, which can then be differentiated into a new cell type. ${ }^{2}$ The inherent regenerative ability of the pancreas, although limited in its postnatal capacity, suggests that there is an endogenous mechanism by which the adult pancreas can develop new $\beta$ cells. This is supported by the previously discussed evidence of differing glycemic conditions correlating with different rates of $\beta$-cell regeneration. ${ }^{17}$ The mature pancreas is composed of 3 cell types: acinar cells, which secrete digestive enzymes; duct cells, which form pathways by which such enzymes are transported into the gastrointestinal tract; and endocrine cells found in the islets of Langerhans. ${ }^{21}$ Glucose homeostasis is dependent on the function of $\alpha$ cells secreting glucagon and $\beta$ cells secreting insulin from these islets. Acinar cells, duct epithelial cells, and $\alpha$ cells have been identified as 3 types of possible progenitor cells capable of postnatal transdifferentiation into $\beta$ cells. ${ }^{2}$ Research focusing on the transdifferentiation of $\alpha$ cells has appeared most promising because $\alpha$ cells are closely related to $\beta$ cells not only in their location, but in their glucose homeostasis role as well. ${ }^{18}$ The ability to harness and transdifferentiate these progenitor cells into bona fide $\beta$ cells could provide a cure for type 1 diabetes and could alleviate severe progression of type 2 diabetes.

The idea of using existing pancreatic cells as progenitors for $\beta$-cell regeneration was introduced by iPSC research that found that human pancreatic duct-derived stem cells had superior $\beta$-cell differentiation potential compared with those derived from human skin fibroblasts. ${ }^{5}$ Research has focused on identifying the unique features of pancreatic cells that make them more effective progenitors of $\beta$ cells than their stem cell-derived counterparts. Additionally, as previously discussed here, iPSCs and ESCs often have intricate differentiation protocols, which can make it difficult to translate research into safe clinical applications for patients with diabetes. The inherent pancreatic lineage and genetic makeup of pancreatic progenitor cells allow transdifferentiation to occur by a much less complex process, therefore providing a more feasible route toward $\beta$-cell regeneration, which could be translated into a clinical application in humans. ${ }^{2}$ Here, we will outline the current methods by which pancreatic progenitor cells have been found to transdifferentiate into $\beta$ cells, as well as address the clinical application of these findings for patients with diabetes.

While diabetes has long been characterized by poor insulin homeostasis, it has also been suggested that abnormal $\alpha$-cell secretion of glucagon plays an important role in its etiology. 18

This is supported by a study by Damond et $\mathrm{a}^{18}$ of a knockdown of a glucagon receptor preventing the development of diabetes under conditions of $\beta$-cell destruction in mice. Their research clarified that the potential inhibition of glucagon receptors as a treatment for diabetes was only feasible when a certain number of residual $\beta$ cells were present, therefore rendering this research 
inapplicable to patients with type 1 diabetes. ${ }^{18}$ This intimate relationship between $\alpha$ and $\beta$ cells in glucose homeostasis and diabetic etiology, however, led to interest in their differentiation association and plasticity. In 2010, genetic lineage tracing first demonstrated the ability of $\alpha$ cells to transdifferentiate into $\beta$ cells in vivo using a mouse model. ${ }^{19}$ Thorel et a ${ }^{19}$ selectively ablated $\beta$ cells with diphtheria toxin and kept the mice alive with insulin administrations as needed. Within 1 month, they observed a 3 -fold increase in the number of $\beta$ cells present; genetic lineage tracing demonstrated $\alpha$ cells as progenitors of these new $\beta$ cells. Transdifferentiation was initially thought to be spontaneous; however, more recent research has demonstrated that the mechanism is intricately regulated. ${ }^{20,27}$ In 2009, the transcription factor Pax4 was identified as inducing mature $\alpha$ cells to adopt a $\beta$-cell destiny. ${ }^{20}$ Collombat et al ${ }^{20}$ demonstrated that Pax4-induced transdifferentiation of $\alpha$ cells into $\beta$ cells resulted in glucagon deficiency, which then stimulated the continuous neogenesis of new $\alpha$ cells. They reported that the proendocrine gene $\mathrm{Ngn} 3$ was re-expressed and transdifferentiation into $\beta$ cells remained continuous as long as Pax 4 was present. Pax 4 acts as an antagonist repressing the homeobox Arx, which is specific to pancreatic $\alpha$ cells. ${ }^{20}$ In 2013, Courtney et al ${ }^{27}$ reported that the selective inhibition of Arx in $\alpha$ cells is sufficient to promote transdifferentiation into $\beta$ cells, regardless of age. Furthermore, this study identified the inhibition of Arx as the main trigger in the $\alpha$-to $\beta$-cell conversion using conditional double mutant analyses, which indicated that Pax4 is dispensable in the mechanism of this conversion. ${ }^{27}$

Additional research has investigated the possible mechanism of epigenetic manipulation in the transdifferentiation of pancreatic islet $\alpha$ cells to $\beta$ cells. $^{28}$ Bramswig et $\mathrm{al}^{28}$ reported that genes in differentiated $\alpha$ cells are bivalently marked by histone modifications that are both activating (H3K4me3) and repressing (H3K27me3), whereas in $\beta$ cells, these genes are in a monovalent state, marked only by the repressing histone modification. These RNA sequence analyses first suggested that the conversion of $\alpha$ to $\beta$ cells may be dependent on the modulation of histone modifications. ${ }^{28}$ Additionally, epigenetic modification via DNA methylation has been associated with the onset of diabetes. ${ }^{29}$ High glucose levels are understood to induce DNA methylation at the cAMP response element of the Ins1 promoter and consequently inhibit the transcription of Ins1 mRNA. ${ }^{29}$ Metformin, a prevalent diabetic therapeutic used in clinics today, targets this promoter to suppress DNA methylation and upregulate insulin gene expression. ${ }^{29}$ Ishikawa et $\mathrm{al}^{29}$ reported that histones associated with the insulin gene in islet cells were observed to be hyperacetylated and less methylated in $\beta$ cells than in other cells that do not express insulin. Furthermore, DNA methylome analysis observed different methylation patterns at 17 other genes in the islets of patients with type 2 diabetes as compared with patients without diabetes. ${ }^{29}$ These findings provide insight into the pathology of type 2 diabetes and confirm that there is 1 or more epigenetic component(s) that modulates the function of $\beta$ cells. 
Furthermore, this research suggests that glucotoxicity could interfere with the transdifferentiation of islet cells into functional $\beta$ cells considering that glucose levels have an epigenetic influence on the expression of insulin secretion genes. Therefore, epigenetics must be considered in the development of transdifferentiation protocols to regenerate $\beta$ cells in patients with diabetes.

Most recently, $\alpha$-cell transdifferentiation into $\beta$ cells has been associated with $\gamma$-Aminobutyric acid (GABA) signaling pathways. ${ }^{22-24}$ In 2014, GABA treatments were first observed to increase proliferation and decrease apoptosis in human $\beta$ cells grafted in mice. ${ }^{22}$ Mice in this study exhibited increased insulin levels, decreased glucagon and blood glucose levels, and ultimately an improved ability to maintain glucose homeostasis. These findings are consistent with research in which a transcriptional screen identified upregulation of genes involved in GABA signaling pathways when Pax4 was overexpressed. ${ }^{21}$ In vivo experiments conducted by Ben-Othman et al ${ }^{21}$ demonstrated that the treatment of $\alpha$ cells with GABA decreased the expression of Arx. Furthermore, continuous GABA treatments spanning 2 months led to a 3-fold increase in $\beta$-cell mass in WT mice. ${ }^{21}$ Genetic lineage tracing detected that most of these new cells were derived from a glucagonpositive progenitor, suggesting $\alpha$ cells as the origin of new $\beta$ cells. Ben-Othman et $\mathrm{al}^{21}$ applied these findings to a diabetic mouse model and were able to demonstrate that GABA treatments reversed $\beta$-cell destruction by conversion of $\alpha$ cells to $\beta$ cells. Recently, $\mathrm{Li}$ et $\mathrm{al}^{27}$ complemented these findings when they discovered that enhancing GABAA receptor signaling could induce transdifferentiation by altering the identity of $\alpha$ cells. The researchers used artemisinin, an antimalarial drug, which binds to the receptor gephryin to enhance GABA signaling. Gephryin has previously been associated with the synthesis of GABA and its receptors. ${ }^{24} \mathrm{Li}$ et al ${ }^{24}$ observed that treatment with artemisinin increased GABA signaling and inhibited Arx expression, and consequently, $\alpha$ cells secreted less glucagon. Furthermore, additional in vivo studies in both zebrafish and mice demonstrated that artemisinin treatments induced $\beta$-cell proliferation, and lineage analyses identified $\alpha$ cells as the origin of the new $\beta$ cells. ${ }^{24}$

The specific pathway by which GABA signaling induces translocation of Arx to the cytoplasm and consequently its repression remains undefined. ${ }^{21}$ Nevertheless, these intriguing findings characterize an exogenous mechanism by which the pancreas can be induced to transdifferentiate $\alpha$ cells into $\beta$ cells. Perhaps the most exciting aspect of these findings is that therapeutics for GABA signaling targets are already approved for clinical use. ${ }^{25}$ For example, GABAA receptor agonists, such as benzodiazepines, are used as clinical therapeutics for patients with epilepsy and artemisinins are used as therapeutics for patients with malaria worldwide. ${ }^{25}$ 
When used in the correct dosage, these GABA receptor agonists pose minimal risks. ${ }^{26}$ Because GABA is an inhibitory neurotransmitter, those minimal risks have the potential to cause sedative effects such as drowsiness, hypotension, or impaired motor function. ${ }^{26}$ These therapeutics are highly regulated, however, and because of their approved safe use in humans, research in the field of GABA-induced transdifferentiation to regenerate $\beta$ cells in patients with diabetes is promising. Moreover, the endogenous ability of $\alpha$ cells to spontaneously replenish in response to low glucagon levels incites transdifferentiation of $\alpha$ cells as a possible unlimited source of $\beta$-cell replenishment. $^{20}$

\section{Conclusion}

Research in pancreatic regeneration of $\beta$ cells has advanced in the fields of stem cell neogenesis, duplication of existing $\beta$ cells, and transdifferentiation of existing pancreatic progenitors. Current research has demonstrated that the use of stem cells in the neogenesis of $\beta$ cells is not without significant shortcomings. The use of ESCs presents ethical concerns and requires immunosuppressants or complex encapsulation techniques due to the difference in genetic material between donor and recipient. The genetic material of iPSCs in the donor and recipient is one and the same, which solves this problem posed by ESCs. However, the use of both ESCs and iPSCs involves tumorigenesis risks; therefore, the clinical use of these stem cells would require severe quality and safety controls.

The regeneration of $\beta$ cells via the duplication of existing $\beta$ cells seems to be a promising target for diabetic therapeutics, given it already occurs in vivo. However, current research into this endogenous mechanism has presented challenges as to how it may be induced in adult patients with diabetes. Glucose is a major mitogenic factor. As discussed, however, administering glucose to induce $\beta$-cell regeneration would be contraindicated in patients with diabetes because an overload of glucose would further exacerbate hyperglycemia. Additionally, although all $\beta$ cells have an equal capability to self-duplicate, they do so very slowly. This rate of duplication further decreases with age. Further, when $\beta$-cell duplication is induced, there is an increased possibility of cell apoptosis and interference with protective mechanisms may induce tumorigenesis. Future directions of research would need to address these challenges in order for $\beta$-cell duplication to be considered a viable therapy for patients with diabetes. Furthermore, $\beta$-cell duplication would be limited to patients with sufficient residual $\beta$ cells, thus excluding most patients with type 1 diabetes and some patients with type 2 diabetes.

Transdifferentiation of pancreatic islet $\alpha$ cells appears to be one of the most promising mechanisms by which $\beta$-cell regeneration can be induced to replace dysfunctional or destroyed $\beta$ cells in patients with diabetes. There are multiple ways in which this process is prompted including the upregulation of Pax4, the selective inhibition of Arx, the modulation of epigenetic factors, and most 
recently the stimulation of GABA signaling pathways. The GABA signaling findings are particularly promising because therapeutics that could potentially exogenously stimulate transdifferentiation of $\alpha$ cells into $\beta$ cells are already approved for safe use in humans. Considering how $\alpha$ cells can spontaneously regenerate in the adult human pancreas in response to low glucagon levels, this promises an unlimited source of $\beta$ cells for replacement in patients with diabetes. $^{20}$

When comparing the 3 main mechanisms of pancreatic regeneration, transdifferentiation appears to offer the most promising route toward discovering a therapeutic that can replace $\beta$ cells in patients with diabetes. First, transdifferentiation does not present ethical concerns. Second, there is no immune response because the progenitor cells are existing pancreatic cells. Third, because transdifferentiation does not require the insertion of foreign genetic material, tumorigenesis risks can be avoided. Additionally, transdifferentiation is preferable to the duplication of existing $\beta$ cells because the supply of $\beta$ cells in patients with type 1 diabetes mellitus is limited and the supply in patients with type 2 diabetes mellitus is residual as well. The process of transdifferentiation of existing pancreatic progenitor cells into $\beta$ cells, although simpler than the process of differentiating ESCs and iPSCs, is influenced by a concert of transcription factors, epigenetic regulations, and responses to systemic stimuli including blood glucose levels, insulin, and glucagon. In applying this research to replace lost $\beta$ cells, particular attention needs to be made to all these factors to ensure the most efficient method by which pancreatic regeneration can be induced. Thus far, the induction of GABA signaling seems to be the most hopeful route of achieving transdifferentiation of existing pancreatic progenitor cells, especially in light of the existence of therapies already approved for safe use in humans targeting this same pathway. Overall, transdifferentiation of pancreatic $\alpha$ cells promises future regeneration of $\beta$ cells in patients with diabetes. 


\section{REFERENCES}

1. World Health Organization. Global Report on Diabetes. Geneva, Switzerland: World Health Organization; 2016.

2. Kim H, Lee M. $\beta$-Cell regeneration through the transdifferentiation of pancreatic cells: Pancreatic progenitor cells in the pancreas.J Diabetes Investig. 2016;7(3):286-296.

3. Barton FB, Rickels MR, Alejandro R, et al. Improvement in outcomes of clinical islet transplantation: 1999-2010. Diabetes Care. 2012;35(7):1436-1445.

4. In't Veld P, De Munck N, Van Belle K, et al. Beta-cell replication is increased in donor organs from young patients after prolonged life support. Diabetes. 2010;59(7):1702-1708.

5. Yu K, Fischbach S, Xiao X. Beta cell regeneration in adult mice: Controversy over the involvement of stem cells. Curr Stem Cell Res Ther. 2016;11(7):542-546.

6. Rezania A, Bruin JE, Arora P, et al. Reversal of diabetes with insulin-producing cells derived in vitro from human pluripotent stem cells. Nat Biotech. 2014;32(11):1121-1133.

7. Vegas AJ, Veiseh O, Gurtler M, et al. Long-term glycemic control using polymer-encapsulated human stem cell-derived beta cells in immune-competent mice. Nat Med.2016;22(3):306-311.

8. Thomson JA, Itskovitz-Eldor J, Shapiro SS, et al. Embryonic stem cell lines derived from human blastocysts. Science. 1998;282(5391):1145-1147.

9. El Khatib MM, Ohmine S, Jacobus EJ, et al. Tumor-free transplantation of patient-derived induced pluripotent stem cell progeny for customized islet regeneration. Stem Cells Transl Med. 2016;5(5):694-702.

10. Zhang D, Jiang W, Liu M, et al. Highly efficient differentiation of human ES cells and iPS cells into mature pancreatic insulin-producing cells. Cell Res. 2009;19(4):429-438.

11. Takahashi K, Narita M, Yokura M, Ichisaka T, Yamanaka S. Human induced pluripotent stem cells on autologous feeders. Pera M, ed. PLoS ONE. 2009;4(12):e8067.

12. Dor Y, Brown J, Martinez OI, Melton DA. Adult pancreatic beta-cells are formed by selfduplication rather than stem-cell differentiation. Nature. 2004;429(6987):41-46.

13. Teta M, Rankin MM, Long SY, Stein GM, Kushner JA. Growth and regeneration of adult $\beta$ cells does not involve specialized progenitors. Dev Cell. 2007;12(5):817-826.

14. Georgia S, Bhushan A. Beta cell replication is the primary mechanism for maintaining postnatal beta cell mass. J Clin Invest. 2004;114(7):963-968.

15. Meier JJ, Butler AE, Saisho Y, et al. Beta-cell replication is the primary mechanism subserving the postnatal expansion of beta-cell mass in humans. Diabetes. 2008;57(6):1584-1594.

16. Hija A, Salpeter S, Klochendler A, et al. G0-G1 transition and the restriction point in pancreatic beta-cells in vivo. Diabetes. 2014;63(2):578-584.

17. Porat $S$, Weinberg-Corem N, Tornovsky-Babaey $S$, et al. Control of pancreatic $\beta$ cell regeneration by glucose metabolism. Cell Metabolism. 2011;13(4):440-449.

18. Damond N, Thorel F, Moyers JS, et al. Blockade of glucagon signaling prevents or reverses diabetes onset only if residual $\beta$-cells persist. Elife. 2016;5. 
19. Thorel F, Népote V, Avril I, et al. Conversion of adult pancreatic $\alpha$-cells to $\beta$-cells after extreme $\beta$-cell loss. Nature. 2010;464(7292):1149-1154.

20. Collombat $\mathrm{P}, \mathrm{Xu} X$, Ravassard P, et al. The ectopic expression of Pax4 in the mouse pancreas converts progenitor cells into $\alpha$ and subsequently $\beta$ cells. Cell. 2009;138(3):449-462.

21. Ben-Othman N, Vieira A, Courtney M, et al. Long-term GABA administration induces alpha cell-mediated beta-like cell neogenesis. Cell. 2017;168(1-2):73-85.e11.

22. Purwana I, Zheng J, Li X, et al. GABA promotes human beta-cell proliferation and modulates glucose homeostasis. Diabetes. 2014;63(12):4197-4205.

23. Weir GC, Bonner-Weir S. GABA signaling stimulates $\beta$ cell regeneration in diabetic mice. Cell. 2017;168(1):7-9.

24. Li J, Casteels T, Frogne T, et al. Artemisinins target GABAA receptor signaling and impair a cell identity. Cell. 2017;168:1-15.

25. Rutter GA. GABA signaling: A route to new pancreatic $\beta$ cells. Cell Res. 2017;27(3):309-310.

26. Julien RM. A Primer of Drug Action: A Concise Nontechnical Guide to the Actions, Uses, and Side Effects of Psychoactive Drugs, Revised and Updated. Henry Holt and Company; 2013.

27. Courtney M, Gjernes E, Druelle N, et al. The inactivation of arx in pancreatic $\alpha$-cells triggers their neogenesis and conversion into functional $\beta$-like cells. Habener J, ed. PLoS Genet.

2013;9(10):e1003934.

28. Bramswig NC, Everett LJ, Schug J, et al. Epigenomic plasticity enables human pancreatic $\alpha$ to $\beta$ cell reprogramming. J Clin Invest. 2013;123(3):1275-1284.

29. Ishikawa $\mathrm{K}$, Tsunekawa $S$, Ikeniwa $M$, et al. Long-term pancreatic beta cell exposure to high levels of glucose but not palmitate induces DNA methylation within the insulin gene promoter and represses transcriptional activity. Ayuso E, ed. PLoS ONE. 2015;10(2):e0115350. 